Advances in Radio Science (2003) 1: 15-20

(C) Copernicus $\mathrm{GmbH} 2003$

\title{
Optimization of traceable coaxial RF reflection standards with 7-mm-N-connector using genetic algorithms
}

\section{T. Schrader}

Physikalisch-Technische Bundesanstalt, Laboratory for High-frequency Measurement Techniques, Bundesallee 100, D-38116 Braunschweig, Germany

\begin{abstract}
A new coaxial device with 7-mm-N-connector was developed providing calculable complex reflection coefficients for traceable calibration of vector network analyzers (VNA). It was specifically designed to fill the gap between $0 \mathrm{~Hz}$ (DC, direct current) and $250 \mathrm{MHz}$, though the device was tested up to $10 \mathrm{GHz}$. The frequency dependent reflection coefficient of this device can be described by a model, which is characterized by traceable measurements. It is therefore regarded as a "traceable model". The new idea of using such models for traceability has been verified, found to be valid and was used for these investigations. The DC resistance value was extracted from $\mathrm{RF}$ measurements up to $10 \mathrm{GHz}$ by means of Genetic Algorithms (GA). The GA was used to obtain the elements of the model describing the reflection coefficient $\Gamma$ of a network of SMD resistors. The DC values determined with the GA from RF measurements match the traceable value at DC within $3 \cdot 10^{-3}$, which is in good agreement with measurements using reference air lines at $\mathrm{GHz}$ frequencies.
\end{abstract}

\section{Introduction}

To obtain transmission and reflection properties of arbitrary RF circuits, vector network analyzers (VNA) are widely used today. Those devices require a calibration to improve the system performance and to reduce errors induced by nonideal components like couplers, cables etc. Over the years a broad range of calibration techniques has been developed for VNAs. Most of them depend on known calibration standards, except those routines which are known as self-calibration procedures. The well-known TMSO or SOLT schemes (T: through, M: match, S: short circuit, O: open circuit, L: line) make use of predictable and characterized items. Even though the calibration items are used to obtain the error-box

Correspondence to: T. Schrader

(Thorsten.Schrader@ptb.de) parameters of theVNA, they do not necessarily provide traceability to national standards.

If these items are used for calibration of VNAs, the measurement uncertainty is of interest as well as the traceability of the results to national standards. Extensive theoretical and experimental investigations have been carried out to obtain e.g. the influence on scattering parameters of non-ideal calibration standards, which are assumed to be error-free like low-band matches (Stumper, 2002; Stumper, 2003). Uncertainty will not be the main topic of this paper except for estimation of the additional uncertainty while applying this method. Here we describe a new method of obtaining traceability of measurements of reflection coefficients at frequencies below $250 \mathrm{MHz}$.

At PTB the traceability of RF complex reflection coefficients is achieved by the cross-ratio method (Stumper, 1993) using calculable transmission line impedance standards (Leuchtmann, 2002) i.e. reference coaxial precision air lines without any supporting beads of the center conductor. For reflection coefficients $\Gamma$ of low-loss air lines, the formulae indicate only the ratio of the inner diameter of the outer conductor $r_{a}$ to the diameter of the center conductor $r_{i}$ as independent input values, therefore $\Gamma$ is traced back to length standards of PTB. The usable frequency range of reference air lines is limited, firstly due to mechanical restrictions in the process of manufacturing and an applicable length of ca. $300 \mathrm{~mm}$ at the largest (for a $7 \mathrm{~mm}$-air line with $\mathrm{N}$-connector) and secondly due to electrical restrictions regarding the characteristic impedance of such lines. Both reasons set the lower frequency limit to about $10 \mathrm{MHz}$, while the upper limit is defined by that frequency which renders a pure TEM transmission mode. Hence, the $7 \mathrm{~mm}$ line may be used up to $18 \mathrm{GHz}$.

It is obvious that the mechanical tolerances, particularly those of the center conductor, have to be kept as small as possible. Claiming an error of $\Gamma$ due to geometry deviations to be less than $5 \cdot 10^{-4}, \delta r_{a}$ and $\delta r_{i}$ are in the order of $1 \mu \mathrm{m}$. Assuming a low-loss air line, the line impedance becomes a real value. For the skin depth being small compared to the radii of the conductors, this can be checked by calculating 


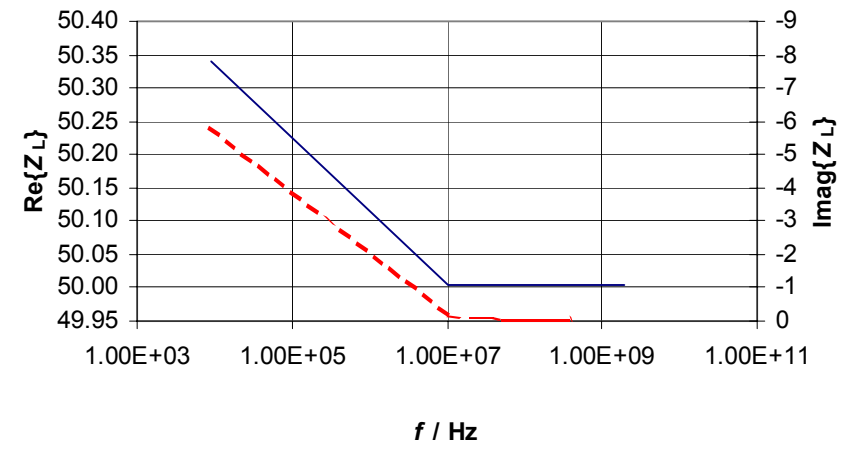

Fig. 1. Real part of characteristic impedance of a low-loss $7 \mathrm{~mm}$ coaxial air line (solid), imaginary part of characteristic impedance (dashed line), calculated for inner and outer conductor made of gold.

the line equivalent elements per unit length $\left(R^{\prime}, G^{\prime}, L^{\prime}, C^{\prime}\right)$. The complex line impedance $Z_{L}$ is calculated using the wellknown equation

$Z_{L}=\sqrt{\left(R^{\prime}+j \cdot \omega \cdot L^{\prime}\right) /\left(G^{\prime}+j \cdot \omega \cdot C^{\prime}\right)}$

with $R^{\prime}$ : series resistance per unit length (p.u.1.), $G^{\prime}$ : shunt conductance p.u.1., $L^{\prime}$ : high-frequency inductance p.u.1., $C^{\prime}$ : high-frequency capacitance p.u.l..

For lossy coaxial transmission air lines the equivalent elements p.u.l. can be calculated by using (Rizzi, 1988)

$G^{\prime}=0$

$R^{\prime} \approx \frac{1}{2 \pi \cdot r_{i} \delta_{i} \sigma_{i}}+\frac{1}{2 \pi \cdot r_{a} \delta_{i} \sigma_{a}}$

$L^{\prime}=\frac{\mu_{0} \mu_{r}}{2 \pi} \cdot \ln \frac{r_{a}}{r_{i}}$

$C^{\prime}=\frac{2 \pi \varepsilon_{0} \varepsilon_{r}}{\ln \left(\frac{r_{a}}{r_{i}}\right)}$

with the skin depths on outer and inner conductor

$\delta_{a}=\frac{1}{\sqrt{\pi \cdot f \cdot \mu_{0} \mu_{r} \sigma_{a}}}, \quad \delta_{i}=\frac{1}{\sqrt{\pi \cdot f \cdot \mu_{0} \mu_{r} \sigma_{i}}}$

with $\mu_{r}, \varepsilon_{r}=1$, the DC conductivities $\sigma_{a}=\sigma_{i}=\sigma_{A u}=$ $4,1 \cdot 10^{7} \mathrm{~S} / \mathrm{m}, r_{a}=7,00024 \cdot 10^{-3} \mathrm{~m}$, and $r_{i}=3,04038$. $10^{-3} \mathrm{~m}$.

The characteristic line impedance vs. frequency is given in Fig. 1. It becomes complex for frequencies below $10 \mathrm{MHz}$. By using the extended cross-ratio method (Stumper, 1993), a worst-case calculation of the expanded uncertainty of the complex reflection coefficient $\Gamma$ of coaxial $7 \mathrm{~mm}$ reference air lines inserted in an ideal $50 \Omega$ coaxial system yields $13,1 \cdot 10^{-2}$ at $1 \mathrm{MHz}, 1,4 \cdot 10^{-2}$ at $10 \mathrm{MHz}, 3,2 \cdot 10^{-3}$ at $100 \mathrm{MHz}$, and $3 \cdot 10^{-3}$ at $250 \mathrm{MHz}$. Alternative methods to determine traceable reflection coefficients in the $\mathrm{MHz}$ range should yield uncertainty values not exceeding $3 \cdot 10^{-3}$.

To provide traceability of reflection coefficients $\Gamma$ to national standards Stumper $(1995 ; 2000)$ presented a method using resistive layers of nichrome filling the cross-section of $7 \mathrm{~mm}$ coaxial transmission lines. The nichrome was sputtered on thin capton foils with gold electrodes providing contact to the outer and inner conductor of the line. The thickness of the resistive layers being kept small against the skin depth, the skin effect does not change the real part of the resistance from DC to at least $2 \mathrm{GHz}$. The imaginary part of $\Gamma$ is determined by the capacitance between inner and outer conductor at the end of the inner conductor. The DC resistance of the layer is traced back to national DC standards, so the complex reflection coefficient is traceable to DC resistance as long as an influence of the skin effect is negligible. It has been shown that short-term stability is given and that the method proves to yield the expected values. The main uncertainty contribution is due to alteration of the DC resistance caused by mechanical stress of the thin layer structure of nichrome on capton foil each time the device was connected to measure the reflection coefficient. Other problems arose from oxidation and drifting effects of the material properties.

\section{New design}

To avoid aging and drifting of the planar resistive layers, SMD (surface mounted device) resistors were used instead. These devices are processed on ceramic substrates using thin film technology with tempering and passivation afterwards. For the frequency range considered, the ceramic substrates are regarded to be loss-less. The well designed mixture of $\mathrm{Ni}$, $\mathrm{Cr}$, and $\mathrm{Al}$ reduces the temperature coefficient to a minimum, while long term stability is obtained by the appropriate manufacturing process. Small sized devices such as standard type HF 0102 or 0204 fit to the $7 \mathrm{~mm}$ coaxial line. To obtain an evenly distributed surface current transition from the conductors to the resistors, inserts consisting of up to eight of these resistors of equal resistance are assembled in such a way that the resistors are arranged symmetrically between center and outer conductor. Eight resistors equally distributed resemble an almost homogeneous resistive layer inside a cross-section of the $7 \mathrm{~mm}$ line. We have also tried two or four resistors, forming angles between 20 and 180 degrees to study coupling, mutual inductance and proximity effects. With the inserts, the resistors are hold in place by soldering joints on copper foil. Therefore, a thin washer was cut from copper foil $30 \mu \mathrm{m}$ in thickness. The outer diameter of the washer fits the corresponding dimension of the line, while the inner diameter is smaller than the inner diameter of the outer conductor, in this way we obtain a diaphragm. A disk cut from copper foil with dimensions slightly larger than the diameter of the center conductor of the $7 \mathrm{~mm}$ line provides a soldering joint at the inner conductor.

The drift rate of resistance with temperature is dependent on the highest temperature the resistor is exposed during the soldering process. In order to keep mechanical and temperature stress as low as possible, Woods material with a decent flow rate at 70 degrees Celsius was used as solder and 
the temperature of the soldering iron was set to nearly 80 degrees Celsius at the most. The inserts are mounted into a special receptacle which was designed for this purpose, adaptable to the $7 \mathrm{~mm}$ coaxial $\mathrm{N}$-connector at the VNA reference plane. The outer conductor of the receptacle clamps the insert when it is screwed together, while the center contact is spring loaded to take mechanical forces during assembly, similar to the design of Stumper (2000). The insert in combination with the receptacle is furthermore mentioned as d.u.t..

\section{Theory}

With the intention to provide traceability of complex reflection coefficients in the frequency range from $\mathrm{DC}$ to $250 \mathrm{MHz}$ we will introduce some new ideas based on what we call "traceable model". The basic ideas are to set up a network of lumped elements as a model, which describes the reflection coefficient. The next step is to link this model with experimental data and to optimize the model according to the experiment. Therefore, the model has to be validated at the experimental sampling points. The actual complex reflection coefficients can now be calculated at any frequency from the model instead of assuming the values. Of course, the experimental data must be traceable at the sampling points to national standards. Here, traceable measurement results can be obtained for $\mathrm{DC}$ and for frequencies above $250 \mathrm{MHz}$, but not in between. To fill this gap, we will rely on the traceable model. If the frequency range increases to $10 \mathrm{GHz}$, the model becomes extremely sensitive against the influence of parasitics. Decreasing the frequency to the $\mathrm{kHz}$ range and down further to zero, it can be shown that this influence drops monotonously to zero as well, as long as not any resonance occurs in the frequency response. This is not the case as such resonances were not observed when measuring the insert at low frequencies after a MSO calibration, using a lowband matched termination taken from the calibration kit of the VNA for the M. From these considerations it can be concluded, that the validated model can provide traceability of reflection coefficients at frequencies below $250 \mathrm{MHz}$.

\subsection{The mode}

The resistive network consists of one SMD resistor or several resistors of (nominal) equal resistance. We have tested up to 8 elements. Each resistor as mounted in the insert can be described by its DC resistance value $R_{a}$, by the non-linear self-inductance $L_{a}$ along its physical length

$L_{a}=L_{0}+L_{1} \cdot f+L_{2} \cdot f^{2}+L_{3} \cdot f^{3}$

and by the capacitance $C_{a}$ across the soldering contacts. By assembling the inserts, additional inductances, losses and mutual inductances are taken into account, as many times as many resistors are included. The soldering diaphragm and the recessed center conductor of the coaxial line form an additional capacitance. Additional inductances $L_{b}, L_{c}$ and a resistor $R_{v}$ describe the losses of the current transition from the resistor to the copper foils via the soldering joints.
The input admittance of a single SMD resistor as mounted in the insert can be calculated by the following equation

$\underline{Y}_{a}=\frac{1}{R_{a}+j \cdot \omega \cdot L_{a}}+j \cdot \omega \cdot C_{a}$

The impedance of the insert is then

$\underline{Z}_{a}=\frac{1}{n} \cdot\left[\frac{1}{Y_{a}}+R_{v}+j \cdot \omega \cdot\left(L_{b}+L_{c}\right)\right]$

where $n$ is the number of equal resistors.

Strictly, this model is only valid if the resistors are mechanically and electrically equal. However, if the deviations are small, neither the performance of the GA nor the results themselves are impaired. If the resistance values are too different, the given setup will not lead to a converging solution and a more detailed circuit description is then required.

The total input impedance of the insert in combination with the receptacle (d.u.t.) is

$\underline{Z}_{i n}=\frac{1}{1 / \underline{Z}_{a}+j \cdot \omega \cdot C_{v}}+R_{v v}+j \cdot \omega \cdot L_{d}$

where $C_{v}$ is a capacitance, $L_{d}$ an inductance, and $R_{v v}$ a resistance due to the squeeze contact between conductors of the receptacle and the insert.

With reference to $Z_{0}=50 \Omega$ we obtain the calculated complex reflection coefficient $\Gamma_{c}$ which will be compared to the measured one:

$\underline{\Gamma}_{c}=\frac{\underline{Z}_{i n}-Z_{0}}{\underline{Z}_{i n}+Z_{0}}$.

\subsection{The Genetic Algorithm}

Over the past years several Genetic Algorithms (GA) have been developed for optimization tasks (Rahmat-Samii and Michielsen, 1999). Generally the GA is a versatile and powerful tool to find a solution to problems where it would be time consuming or even not possible to calculate the solution straightforwardly. Especially in case of highly multimodal problems the GA is of advantage when a global maximum is to be determined even in the presence of local maxima. However, it should be noticed as well, that applying GA does not guarantee the exact solution, though it can be often shown, that a solution found will be close to the exact one. To find a solution the GA uses processes adopted from nature evolution formalisms. The Darwinian theory of survival of the fittest, jump and creep mutation and mating may be mentioned here. Further details of the functionality can be found in the references. (Goldberg, 1989; Carroll, 1996; RahmatSamii and Michielsen, 1999). In this paper, only those aspects will be given, which are necessary to follow the procedure.

An easy to adopt code may be received from Carroll (1996), which was the basis for the code devised for these investigations. In general, the calculations can be written to find minima or maxima. Starting with GA, the input parameters have to be specified, e.g. the range of definition should 


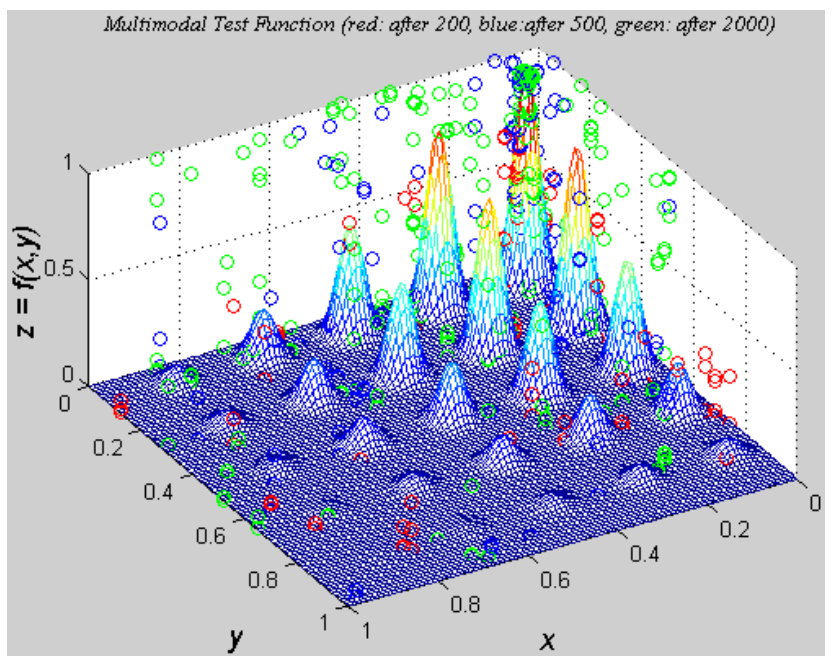

Fig. 2. Test function by (Goldberg and Richardson, 1987) for two independent parameters varied between zero and one. The GA optimizes the solution to the global maximum of the function. Circles indicate performance vs. number of generation (with color varying from red to blue to green).

be given in absolute values and the resolution in steps, respectively. A $2^{n}$ value for the number of steps is recommended. Furthermore, the number of generations and the population size per generation have to be defined in the "inifile". Now we need to define the cost function $d$, with which we calculate the score of the actual settings of our parameters. Here, we compare two complex reflection coefficients, one measured as a function of frequency $\underline{\Gamma}_{m}$, the other calculated as a frequency response $\underline{\Gamma}_{c}$ with the actual settings of parameters, both at the same sampling points. The cost function $d$ is calculated according to Eq. (12), where the absolute value of the complex difference of both reflection coefficients, which is then summed over frequency, determines the deviation of the describing model from the measurement.

$d=1-\sum_{i=f_{u}}^{f_{0}} \sqrt{\left(\operatorname{Re}\left[\underline{\Gamma}_{c, i}-\underline{\Gamma}_{m, i}\right]\right)^{2}+\left(\operatorname{Im}\left[\underline{\Gamma}_{c, i}-\underline{\Gamma}_{m, i}\right]\right)^{2}}$.

Before the optimization starts, the parameters are converted into a binary representation, so that bits may be shuffled, exchanged, mated, etc. The first few sets of parameters are then chosen at random from the range of definition of parameters, the cost function is calculated and from there the GA starts. The best parameter set (yielding the highest cost function $d$ ) is always kept across all generations ("survival of the fittest"), the best parents are mated and again, some new sets are chosen at random. The convergence is increased, if the first estimate of all parameters is in the order of the correct value. Depending on the settings of the GA, more or fewer generations are needed to maximize the cost function.

A suitable test function for the GA was given by Goldberg and Richardson (1987). Figure 2 displays the test function as a 3-D plot, dependent on two parameters $\mathrm{x}$ and $\mathrm{y}$, which are varied between zero and one. The circles, changing their

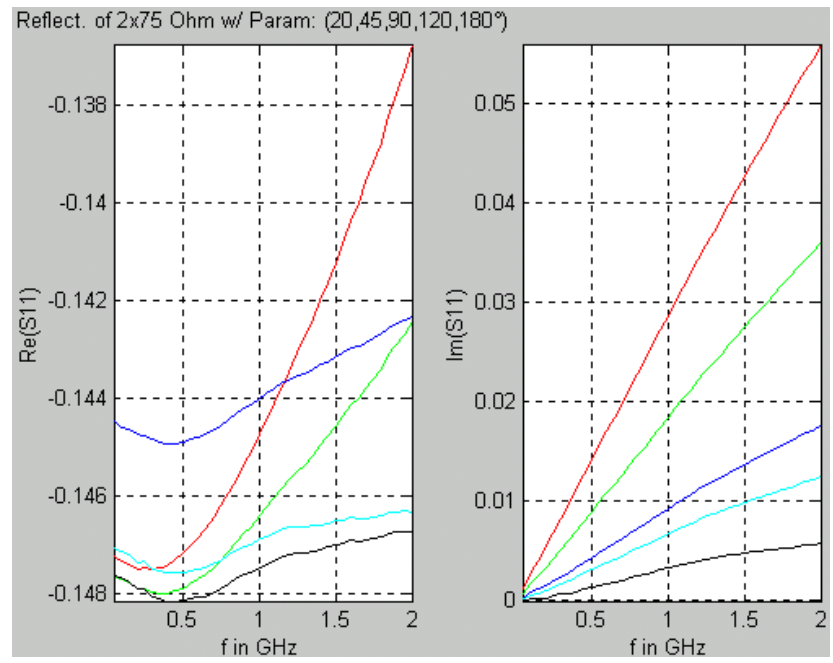

Fig. 3. Measured reflection coefficient of two SMD resistors HF 0102 in parallel, $75 \Omega$ each, mounted in a receptacle with the angle between as parameter. Red: $20^{\circ}$, green: $45^{\circ}$, blue: $90^{\circ}$, black: $120^{\circ}$, light blue: $180^{\circ}$.

color from red over blue to green, mark the main sampling points of the GA and, once a good approximation of the exact solution is obtained, the concentration to the global maximum can be seen.

Usually we stopped the GA process, when a value of the cost function of 0.999 had been achieved. If more accuracy is needed, a second run may be implemented with optimized ranges of definition of the parameters. The "creeping algorithm" implemented in the GA routine should prevent the solution to stop before the "real hill" is reached.

\section{Experimental}

Using an HP $8510 \mathrm{C}$ VNA a regular one-port calibration was performed with the calibration firmware. As calibration standards we have used short and open circuits, sliding load and a low-band matched termination from the VNA calibration kit, the latter for comparison purposes below $2 \mathrm{GHz}$, which is the lower frequency limit for application of the sliding load. In the frequency range from $2 \mathrm{GHz}$ up to $10 \mathrm{GHz}$ the sliding load may be regarded as a traceable device. As described later, a coaxial reference air line with a length of $125 \mathrm{~mm}$ is used as a traceable device down to $600 \mathrm{MHz}$. The DC resistance of the d.u.t. was traced back to DC resistance of PTB with a value of $R_{\text {reflx,meas }}=49,819 \Omega$.

Applying the GA to the RF measurement data we obtain a DC resistance of $R_{\text {reflx }, G A}=49,700 \Omega$. The relative deviation of $R_{r e f l x, G A}$ and $R_{\text {reflx, meas }}$ equals $-2,4 \cdot 10^{-3}$, which is a reasonably good agreement, considering the measurement uncertainties of $2 \cdot 10^{-3}$ for measuring complex RF reflection coefficients using sliding loads for calibration.

Figure 3 shows the influence of coupling between the resistors on the reflection coefficient. Both the real and imag- 


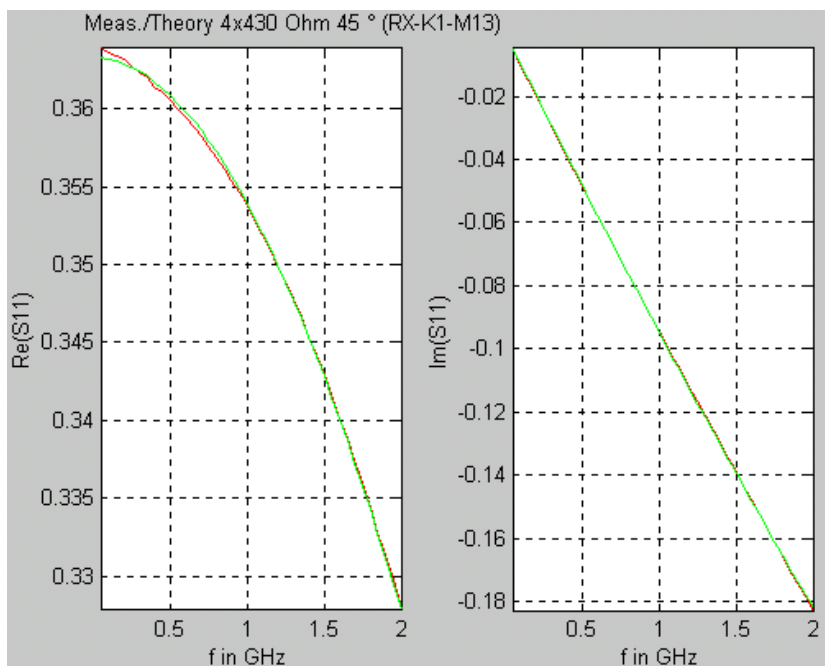

Fig. 4. Real and imaginary part of reflection coefficient of insert with 4 resistors HF 0102 in parallel with 180 ? each, red line: measurement, green: calculated from retrieved model.

inary part are changed by varying the angle formed by the resistors. With two resistors of type HF 0102 mounted in an angle of around $140^{\circ}$, the frequency response of the complex reflection coefficient can be flattened. In spite of the same fabrication process, the resistors, regarding their nominal DC resistance value, show different behavior in complex impedance which can be described as inductive for values below $100 \Omega$ and as capacitive for values above. In Fig. 4 the comparison of the reflection coefficient obtained by measurement and by calculation using the retrieved model is depicted for an insert containing four resistors. While the data shown so far was obtained using an HP 8510 VNA, we have also used an R\&S ZVR VNA down to $9 \mathrm{kHz}$, while its upper frequency limit is $4 \mathrm{GHz}$. The results given in Fig. 5 are an example for measurements utilizing the ZVR. As can be seen from the results in Fig. 5, the solution still remains satisfactory and the retrieved set of parameters describes the measurements very well. The insert contained one resistor of type HF 0102 with a nominal DC resistance of $50 \Omega$. Decreasing the frequency down to the $\mathrm{kHz}$ range, both $\operatorname{Re}\{\Gamma\}$ and $\operatorname{Im}\{\Gamma\}$ should drop down to zero, if the d.u.t. is matched to the characteristic impedance of the line system. In Fig. 5 we encountered $\operatorname{Re}\{\Gamma\}$ to under-run zero. The reason is the DC reference value of the low-band matched termination of the VNA calibration kit. It was determined to $R_{\text {low-band,meas }}=50,087 \Omega$, but the traceable DC resistance of the d.u.t. amounts to $R_{\text {reflx } x \text {,meas }}=49,819 \Omega$. Therefore, $\operatorname{Re}\{\Gamma\}$ should be equal to $-2,68 \cdot 10^{-3}$, which is the case in Fig. 5.

Traceability is given at frequencies above $0,6 \mathrm{GHz}$. Using the cross-ratio method at quarter wavelengths $(\mathrm{QW})$ (Stumper, 1993) with a $125 \mathrm{~mm}$ air line with slotless inner contacts, and comparing the results obtained with this method with the results from measurements using the regular sliding load (s.l.) calibration, only minor deviations of up

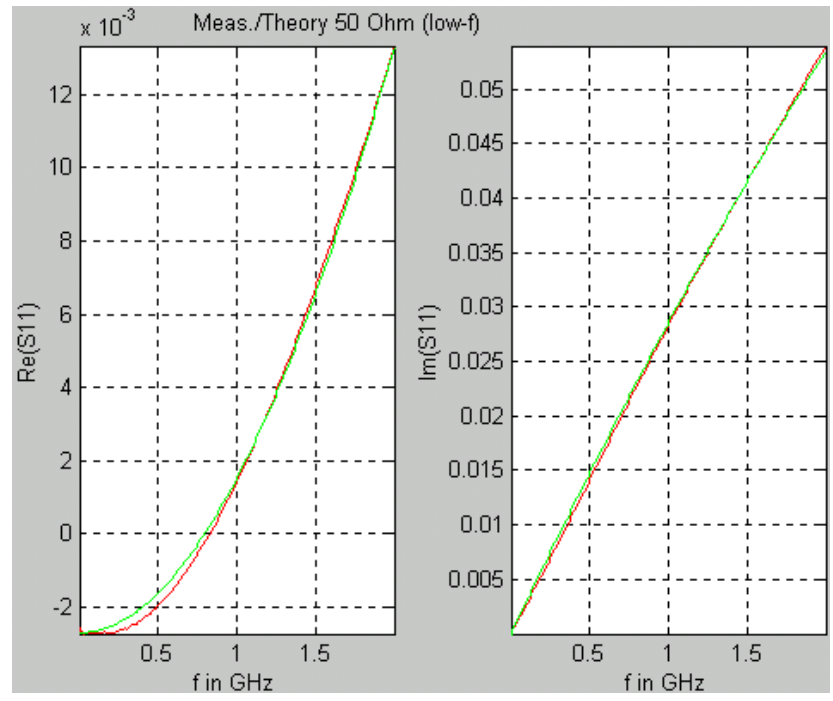

Fig. 5. Real and imaginary part of reflection coefficient of inset with one resistor HF 0102 of nominal $50 \Omega$ resistance (high resolution for $\left.\operatorname{Re}\left\{S_{11}\right\}\right)$. Red line: measurement, green line: calculated from retrieved model.

to $5 \cdot 10^{-3}$ are found at quarter wavelength frequencies. Table 1 gives these deviations for both real and imaginary part of $\Gamma$.

\section{Conclusion}

It has been experimentally verified for a $7 \mathrm{~mm}$ coaxial line, that the RF reflection coefficient can be obtained traceably even for the frequency range between DC and $250 \mathrm{MHz}$. With the new approach of a traceable model we can fill the gap of calculable standards for complex RF reflection coefficients. It can be stated, that the traceable model describes the measurement data up to $10 \mathrm{GHz}$ with negligible deviations, and that the Genetic Algorithm performs very well the matching of calculated and measured data. Exploiting the GA, it was possible to extract the DC resistance of the d.u.t. from RF measurements. The result of the extracted DC resistance value is within the postulated range of uncertainty and, compared to the traceably measured DC resistance value, the relative deviation is less than $-2,4 \cdot 10^{-3}$.

As input data for the GA we have used results obtained from measurements traced either to PTB's DC resistance values and, at RF, to sliding load or reference line impedance standards known as air lines. Hence, the model is verified with data from measurements traceable to national standards. Since it holds for the critical frequency range up to $10 \mathrm{GHz}$ and it shows good agreement for the DC values as well, it can be concluded that the model holds the traceability of complex reflection coefficients for the frequency range in between.

We will use the characterized d.u.t. (instead of the lowband matched termination up to $2 \mathrm{GHz}$ ) for an external calibration procedure to compare with results obtained by the extended cross-ratio method down to about $100 \mathrm{MHz}$. 
Table 1.

\begin{tabular}{ccccccccc}
\hline Freq. & \multicolumn{2}{c}{$50 \mathrm{Ohm}$} & \multicolumn{2}{c}{$2 \times 75$ Ohm } & \multicolumn{2}{c}{$2 \times 91$ Ohm } & \multicolumn{2}{c}{$4 \times 180 \mathrm{Ohm}$} \\
$\mathrm{GHz}$ & $\mathrm{X}(\mathrm{QW}-\mathrm{s} .1)$. & $\mathrm{Y}(\mathrm{QW}-\mathrm{s} .1)$. & $\mathrm{X}(\mathrm{QW}-\mathrm{s} .1)$. & $\mathrm{Y}(\mathrm{QW}-\mathrm{s} .1)$. & $\mathrm{X}(\mathrm{QW}-\mathrm{s} .1)$. & $\mathrm{Y}(\mathrm{QW}-\mathrm{s} .1)$. & $\mathrm{X}(\mathrm{QW}-\mathrm{s} .1)$. & $\mathrm{Y}(\mathrm{QW}-\mathrm{s} .1)$. \\
\hline 0,6 & 0,001 & 0,001 & 0,001 & 0,001 & 0,001 & 0,001 & 0,000 & 0,000 \\
1,8 & 0,001 & 0,000 & 0,000 & 0,000 & 0,000 & 0,000 & $-0,001$ & 0,000 \\
3,0 & $-0,001$ & $-0,001$ & 0,000 & 0,002 & 0,000 & 0,000 & $-0,001$ & 0,000 \\
4,2 & $-0,002$ & 0,001 & 0,001 & 0,000 & $-0,001$ & $-0,001$ & $-0,001$ & $-0,001$ \\
5,4 & 0,000 & 0,003 & 0,000 & 0,000 & $-0,002$ & $-0,001$ & $-0,002$ & $-0,003$ \\
6,6 & 0,002 & 0,002 & 0,000 & 0,002 & $-0,002$ & 0,000 & $-0,003$ & $-0,001$ \\
7,8 & 0,003 & 0,002 & 0,003 & 0,003 & $-0,001$ & 0,002 & $-0,004$ & $-0,001$ \\
9,0 & 0,004 & $-0,001$ & 0,003 & $-0,001$ & $-0,001$ & 0,000 & $-0,005$ & 0,001 \\
10,2 & $-0,001$ & $-0,004$ & 0,001 & $-0,004$ & $-0,002$ & 0,000 & $-0,005$ & 0,000 \\
\hline
\end{tabular}

Acknowledgements. Many thanks to Nick Ridler, National Physical Laboratory, London, England for lending the air line to PTB for the experimental investigations. Special thanks go to Ulrich Stumper (PTB) for many valuable and helpful discussions, to Wolfgang Peinelt (PTB) for providing calibration of the air line and to Ahmad Daneschnejad (PTB) for assembling the inserts.

\section{References}

Stumper, U.: Influence of calibration uncertainties on VNA Sparameter measurements, CPEM'2002 Digest, Conf. on Precision Electromagn. Meas., Ottawa, Canada, pp.132-133, 2002.

Stumper, U.: Influence of TMSO calibration standards uncertainties on VNA S-parameter measurements, IEEE Trans. on Instrum. Meas., 52, in print April, 2003.

Stumper, U.: Determination of Mismatch Factor with RF Power Calibration Using Air line Impedance Standards, IEEE Trans.on Instrum. Meas., 46, pp. 531-534, April 1997.

Leuchtmann , P.: On the Calculation of Air Lines, Kleinheubacher Berichte, 45, pp. 1-5, 2002.

Stumper, U.: Correction of the RF Complex Reflection Coefficient Using Air Line Impedance Standards, IEEE Trans. on Instrum. Meas., 42, pp. 516-518, 1993.
Rizzi, P.: Microwave Engineering, Passive Circuits, Prentice Hall, Englewood Cliffs, New Jersey, ISBN 0-13-586702-9, p.185, 1988.

Stumper, U.: Reflectometer Calibration Using Planar NiCr ThinFilm Resistors and an Open Circuit. IEEE Transactions on Instrumentation and Measurement, 44, pp. 288-290, 1995.

Stumper, U.: Reflektometerkalibrierung mit Dnnfilmwiderstnden in $7 \mathrm{~mm} / 50 \Omega$ - Koaxialtechnik mit N-Konnektor bis $2 \mathrm{GHz}$, Kleinheubacher Berichte, 43, pp. 155-161, 2000.

Rahmat-Samii, Y., and Michielsen, E.: Electromagnetic Optimization by Genetic Algorithms, John Wiley \& Sons, Hoboken, NJ, ISBN: 0471295450, 1999.

Goldberg, D. E.: Genetic Algorithms in Search, Optimization and Machine Learning, Addison-Wesley, Reading, MA, 1989.

Carroll, D. L.: Genetic Algorithms and Optimizing Chemical Oxygen Iodine Lasers, in: Developments in Theoretical and Applied Mechanics, Vol. XVIII, edited by H. Wilson et al. (School of Engineering, The University of Alabama, Alabama, 1996), pp.411424, 1996.

Carroll, D. L.: Chemical Laser Modeling with Genetic Algorithm, AIAA Journal, 34, pp. 338-346, 1996.

Goldberg, D. E. and Richardson, J.: Genetic Algorithms with Sharing for Multimodal Function Optimization, Genetic Algorithms and their applications: Proceedings of the Second International Conference on Genetic Algorithms, pp. 41-49, 1987. 